\title{
DUALITY IN GENERALIZED LINEAR FRACTIONAL PROGRAMMING*
}

\author{
Jean-Pierre CROUZEIX
}

Département de mathématiques appliquées. Université de Clermont. Aubière. France

\author{
Jacques A. FERLAND** \\ Département d'injormatique et de recherche opérationelle, Université de Montréal, Montréal, $P Q$ \\ Canada
}

\author{
Siegfried SCHAIBLE*** \\ Deparment of Finance and Management Science. University of Alberta. Edmonton. AB. Canada
}

Received 24 June 1981

Revised manuscript received 7 February 1983

We consider a generalization of a linear fractional program where the maximum of finitels many linear ratios is to be minimized subject to linear constraints. For this Min-Max problem a dual in the form of a Max-Min problem is introduced and duality relations are established.

Key words: Fractional Programming, Quasiconvex Programming, Duality.

\section{Introduction}

This paper deals with the following nonlinear program

$$
\text { Inf }\{r(x) \mid x \in K\}
$$

where the objective function $r$ is defined as the maximum of $p$ ratios, i.e.

$$
r(x)=\operatorname{Max}_{1 \leq i \leq p} \frac{f_{i}(x)}{g_{i}(x)} .
$$

This is a generalization of a fractional programming problem $(p=1)$ which has been investigated quite actively in the last two decades [21]. In [20] many of the results in fractional programming are reviewed and extended. An extensive bibliography is given in [22].

An early application of generalized fractional programming $(p>1)$ is von Neumann's model of an expanding economy [25]. Here the functions $f_{i}, g_{i}$ are linear and $K$ is the nonnegative orthant.

\footnotetext{
* This work was supported by a Research Grant from NATO, No. 1934.

** Additionally supported by grant NSERC, A8312.

*** Additionally supported by grant NSERC, A4534.
} 
Generalized fractional programs are also encountered in discrete rational approximation where the Chebychev norm is used [2].

Furthermore, generalized fractional programs arise in goal programming where a decision maker wishes to bring several ratios as close as possible to certain predetermined values [13]. The individual goal functions are usually ratios of economic and/or technical terms such as revenue, cost, profit, time, amounts, etc. More details on the applications of fractional programming are given in [20,21]. A goal program involving ratios gives rise to a generalized fractional program if the Chebychev norm is used, as discussed in [4]. Charnes and Cooper [4] stress that this norm has a natural appeal for problems of equity or equality. This is demonstrated, for example, by Vogt's development of an 'Equal Employment Opportunity Index' [24] and by the problem of allocating state funds to educational institutions as discussed by Charnes, Cox and Lane [5].

Problem (1.1), (1.2) is also encountered in multicriteria programming where several ratios are to be optimized simultaneously and the overall objective is to minimize (maximize) the largest (smallest) of these ratios. An application of multicriteria fractional programming is discussed by Ashton and Atkins in [1]. The authors consider ratios that are used in financial planning such as liquidity, return on capital, earnings cover, dividend cover and earnings per share.

Let us now return to the model (1.1), (1.2). If $K$ is a convex set, $f_{i}$ and $-g_{i}$ are convex functions, and either $f_{i}$ is nonnegative and $g_{i}$ positive, or $g_{i}$ is affine and positive, then the objective function $r(x)$ is strictly quasiconvex since it is the maximum of strictly quasiconvex functions. Therefore a local minimum of problem (1.1) is global [14].

If only one ratio appears in (1.2), then a convex-concave fractional program (1.1) can be reduced to a convex program [18]. This is achieved by a generalization of a variable transformation introduced in linear fractional programming by Charnes and Cooper [3]. By reducing (1.1) to a convex program, duality results can be obtained for convex-concave fractional programs $[17,18,19]$. Unfortunately generalized fractional programs $(p>1)$ do not seem to be convex transformable in general, and therefore access to convex duality does not exist.

The first duality results for generalized fractional programs $(p>1)$ were given by J. von Neumann [25] in his paper on an expanding economy. He considers the linear case of $(1.1)$ where $f_{i}, g_{i}$ are linear and $K$ is the nonnegative orthant. A treatment of this model using recent quasiconvex duality results can be found in Crouzeix $[6,7]$ who also investigated the case where $K$ is a compact and convex set, $f_{i}$ are convex and nonnegative and $g_{i}$ are concave and positive. Among other authors who have studied generalized fractional programs we mention Rubinshtein [16] who examined a special linear fractional program using a geometric concept of duality, Gol'stein [10] who examined the case where $K$ is compact and convex, $f_{i}$ are convex and nonnegative and $g_{i}$ are affine using saddle-point results, Passy and Keslassy [15] who investigated certain fractional programs using duality results based on a generalization of Legendre's transformation, and Flachs [9] who 
developed a saddle point duality based on properties of quasiconvex functions $\varphi(x, t)$ which are monotone in $t$. Very recently Jagannathan and Schaible [12] obtained duality relations for (1.1) using Farkas' Lemma.

This paper is concerned with the special case where $K$ is a polyhedral set, $f_{i}$ and $g_{i}$ are affine and $g_{i}$ are positive on $K$. The derivation of a dual program in Section 2 is similar to the one used by Crouzeix [7] for generalized fractional programs. Here the compactness assumption on $K$ is replaced by the linear structure of the program. Notice that this polyhedral structure has been also used by Flachs [9] who derives duality results for similar programs in a different way. In Section 3, we introduce an associated parametric program. Since no compactness assumption is required, the optimal value $v(\mathrm{P})$ of program $(\mathrm{P})$ is not necessarily finite, and the primal and the dual programs have not necessarily optimal solutions. When $v(\mathrm{P})$ is finite, we show in Section 4 that the dual, in contrast to the primal, has always optimal solutions. Conditions to have optimal solutions for the primal are also given. Then complementary slackness is analyzed. Infiniteness of $v(\mathrm{P})$ is studied in Section 5. Finally a special case is considered in Section 6.

Throughout the paper, $A$ and $B$ are two $p \times n$ matrices, $C$ is a $m \times n$ matrix, $\alpha$ and $\beta$ are two $p$-dimensional vectors, and $\gamma$ is an $m$-dimensional vector. For two vectors $x, y$ of the same dimension $\langle x, y\rangle$ denotes their scalar product. We denote by $a_{i}$. (respectively $b_{i}$.) the row $i$ of $A$ (respectively $B$ ) and by $a_{. j}$ (respectively $b_{. j}$ ) the column $j$ of $A$ (respectively $B$ ). We are concerned with the minimization problem

$$
v(\mathrm{P})=\operatorname{Inf}\{r(x) \mid x \in K\}
$$

where

$$
K=\left\{x \in \mathbb{R}^{n} \mid x \geqslant 0, C x \leqslant \gamma\right\}
$$

and

$$
r(x)=\operatorname{Max}_{i \leq i \leq p} \frac{\left\langle a_{i}, x\right\rangle+\alpha_{i}}{\left\langle b_{i}, x\right\rangle+\beta_{i}},
$$

under the assumptions

$\left(\mathrm{H}_{1}\right)$ (Feasibility assumption): there exists $\bar{x} \geqslant 0$ such that $C \bar{x} \geqslant \gamma$, and

$\left(\mathrm{H}_{2}\right)$ (Positivity assumption): $B x+\beta>0$ for all $x \geqslant 0$ such that $C x \leqslant \gamma$.

In some parts of the paper $\left(\mathrm{H}_{2}\right)$ will be replaced by the stronger assumption

$\left(\mathrm{H}_{3}\right)$ (Strong positivity assumption): $b_{i j} \geqslant 0$ for all $i$ and $j$ and $\beta_{i}>0$ for all $i$.

\section{Derivation of a dual problem}

Due to the positivity assumption, an equivalent formulation of $(\mathrm{P})$ is as follows:

$$
v(\mathrm{P})=\operatorname{Inf}_{x, \lambda}\{\lambda \mid A x+\alpha \leqslant \lambda(B x+\beta), x \in K\} .
$$


As in [7], we introduce the following two functions

$$
\varphi(x, u)= \begin{cases}\operatorname{Inf}\{\lambda \mid A x+\alpha+u \leqslant \lambda(B x+\beta)\} & \text { if } x \in K \\ +\infty & \text { otherwise }\end{cases}
$$

and

$$
h(u)=\operatorname{Inf}_{x} \varphi(x, u)
$$

Then a quasiconcave dual program is given as follows:

(D)

$$
v(\mathrm{D})=\operatorname{Sup}_{\lambda, u^{*} \neq 0}\left\{\lambda \mid \xi_{\lambda}\left(u^{*}\right)<0\right\}
$$

where

$$
\xi_{\lambda}\left(u^{*}\right)=\operatorname{Sup}_{x, u}\left\{\left\langle u, u^{*}\right\rangle \mid \varphi(x, u) \leqslant \lambda\right\}
$$

There is no duality gap, i.e. $v(\mathrm{P})=v(\mathrm{D})$ if $h$ is quasiconvex and lower semicontinuous at 0 as shown by Crouzeix $[6,7]$.

It follows from the positivity assumption that the level sets $S_{\lambda}(\varphi)=$ $\{(x, u) \mid \varphi(x, u) \leqslant \lambda\}$ of function $\varphi$ are polyhedral. Indeed it can easily be seen that

$$
S_{\lambda}(\varphi)=\{(x, u) \mid A x+\alpha+u \leqslant \lambda(B x+\beta), x \geqslant 0, C x \leqslant \gamma\} .
$$

The next lemma indicates an important relationship between the level sets of $h$ and those of $\varphi$.

Lemma 2.1. Let the function $f: \mathbb{R}^{r} \times \mathbb{R}^{q} \rightarrow \overline{\mathbb{R}}$, and define the function $g: \mathbb{R}^{r} \rightarrow \overrightarrow{\mathbb{R}}$,

$$
g(x)=\operatorname{Inf}_{y}\{f(x, y)\}
$$

Then for the level sets of $g$ we have $S_{\mu}(g)=\bigcap_{\nu>\mu} \operatorname{Proj}_{R}\left[S_{\nu}(f)\right]$.

Proof. Let $\mu_{1}<\mu_{2}$. Then

$$
\left\{x \mid \text { there exists a } y \dot{\in} \mathbb{R}^{a} \text { such that } f(x, y) \leqslant \mu_{1}\right\} \subset\left\{x \mid g(x) \leqslant \mu_{1}\right\}
$$

and

$$
\left\{x \mid g(x) \leqslant \mu_{1}\right\} \subset\left\{x \mid \text { there exists a } y \in R^{a} \text { such that } f(x, y) \leqslant \mu_{2}\right\} .
$$

Hence

$$
\underset{R^{r}}{\operatorname{Proj}}\left[S_{\mu_{1}}(f)\right] \subset S_{\mu_{1}}(g) \subset \underset{R^{r}}{\operatorname{Proj}}\left[S_{\mu_{2}}(f)\right] .
$$

But $S_{\mu}(f)=\bigcap_{\nu>\mu} S_{\nu}(f)$. Thus

$$
S_{\mu}(g)=\bigcap_{\nu>\mu} \operatorname{Proj}\left[S_{\nu}(f)\right]
$$


We now return to our problem. Since $S_{v}(\varphi)$ is a polyhedral convex set, $\operatorname{Proj}_{R^{r}}\left[S_{\nu}(\varphi)\right]$ is also a polyhedral convex set. Hence $\operatorname{Proj}_{R^{r}}\left[S_{\nu}(\varphi)\right]$ is closed and convex. Applying Lemma 2.1 to $\varphi$ and $h$, it follows that $S_{\mu}(h)=\bigcap_{\nu>\mu} \operatorname{Proj}_{R^{r}}\left[S_{\nu}(\varphi)\right]$ is closed and convex. Thus $h$ is quasiconvex and lower semicontinuous, and hence there is no duality gap, i.e. $v(\mathrm{P})=v(\mathrm{D})$.

Now, we are left to study the functions $\xi_{\lambda}$.

$$
\xi_{\lambda}\left(u^{*}\right)=\sup _{x, u}\left[\left\langle u, u^{*}\right\rangle \mid A x+\alpha+u \leqslant \lambda(B x+\beta), x \geqslant 0, C x \leqslant \gamma\right] .
$$

It follows from the feasibility assumption that $\xi_{\lambda}\left(u^{*}\right)>-\infty$ for all $\lambda$ and $u^{*}$. Furthermore, it is easy to see that, for all $\lambda, \xi_{\lambda}\left(u^{*}\right)=+\infty$ if $u^{*}$ has a negative component. Hence

$$
v(\mathrm{D})=\operatorname{Sup}_{\left.u^{*} \geqslant 0, u^{*} \neq 0\right)}\left\{\operatorname{Sup}_{\lambda}\left\{\lambda \mid \xi_{\lambda}\left(u^{*}\right)<0\right\}\right\} .
$$

Let $u^{*} \geqslant 0, u^{*} \neq 0$, and assume that $\xi_{\lambda}\left(u^{*}\right)$ is finite. The program defining $\xi_{\lambda}\left(u^{*}\right)$ is linear and has at least one optimal solution $(\bar{x}, \bar{u})$. Let $\mu>\lambda$. Then

$$
\xi_{\mu}\left(u^{*}\right) \geqslant\left\langle\bar{u}, u^{*}\right\rangle+(\mu-\lambda)\left(B \bar{x}+\beta, u^{*}\right\rangle,
$$

thus $\xi_{\mu}\left(u^{*}\right)>\xi_{\lambda}\left(u^{*}\right)$. Hence it follows that, for $u^{*} \geqslant 0$,

$$
\operatorname{Sup}_{\lambda}\left\{\lambda \mid \xi_{\lambda}\left(u^{*}\right)<0\right\}=\operatorname{Sup}_{\lambda}\left\{\lambda \mid \xi_{\lambda}\left(u^{*}\right) \leqslant 0\right\} .
$$

Now it is easily seen that, for $u^{*} \geqslant 0$,

$$
\left.\xi_{\lambda}\left(u^{*}\right)=\left\langle\lambda \beta-\alpha, u^{*}\right\rangle+\operatorname{Sup}_{x}\left\{\left\langle\lambda B^{\mathrm{T}}-A^{\mathrm{T}}\right) u^{*}, x\right\rangle \mid x \geqslant 0, C x \leqslant \gamma\right\}
$$

and by linear programming duality

$$
\xi_{\lambda}\left(u^{*}\right)=\left\langle\lambda \beta-\alpha, u^{*}\right\rangle+\operatorname{Inf}_{w^{*} \geqslant 0}\left\{\left\langle\gamma, w^{*}\right\rangle \mid\left(\lambda B^{\mathrm{T}}-A^{\mathrm{T}}\right) u^{*} \leqslant C^{\mathrm{T}} w^{*}\right\} .
$$

When $\xi_{\lambda}\left(u^{*}\right)$ is finite the infimum is reached, and $\xi_{\lambda}\left(u^{*}\right) \leqslant 0$ if and only if there exists a $w^{*} \geqslant 0$ such that $\left\langle\lambda \beta-\alpha, u^{*}\right\rangle+\left\langle\gamma, w^{*}\right\rangle \leqslant 0$ and $\left(\lambda B^{\mathrm{T}}-A^{\mathrm{T}}\right) u^{*}-C^{\mathrm{T}} w^{*} \leqslant 0$. Coming back to the definition of $(D)$, we obtain the dual program

$$
\begin{gathered}
v(\overline{\mathrm{D}})=\operatorname{Sup}_{\lambda, u^{*}, w^{*}}\left\{\lambda \mid\left\langle\lambda \beta-\alpha, u^{*}\right\rangle+\left\langle\gamma, w^{*}\right\rangle \leqslant 0,\left(\lambda B^{\mathrm{T}}-A^{\mathrm{T}}\right) u^{*}-C^{\mathrm{T}} w^{*} \leqslant 0,\right. \\
\left.u^{*} \geqslant 0, w^{*} \geqslant 0, u^{*} \neq 0\right\}
\end{gathered}
$$

with $v(\mathrm{P})=v(\mathrm{D})=v(\overline{\mathrm{D}})$ under assumptions $H_{1}$ and $H_{2}$.

For the single ratio problem $(p=1)$ the dual $(\bar{D})$ was introduced in $[18]$ in a different approach.

To investigate the relationship between $(P)$ and $(\bar{D})$ a related parametric program is analyzed. 


\section{An associated parameteric problem}

Let us consider the following problem

$$
F(\lambda)=\operatorname{Inf}_{x, t}\{\langle 0, x\rangle+t \mid(A x+\alpha)-\lambda(B x+\beta) \leqslant t e, C x \leqslant \gamma, x \geqslant 0\}
$$

where $e=[1,1, \ldots, 1]^{\mathrm{T}} \in \mathbb{R}^{p}$. Notice that $\mathrm{H}_{2}$ implies that $F$ is nonincreasing. In the next theorem, properties of the function $F$ are derived. For the special case of one ratio ( $p=1$ ) $F$ was investigated in $[8,11]$ for a compact feasible region.

Theorem 3.1. If assumptions $\mathrm{H}_{1}$ and $\mathrm{H}_{2}$ hold, then

(i) $F(\lambda)<+\infty$ for all $\lambda \in \mathbb{R}$;

(ii) if $F(\lambda)>-\infty$, then $\lambda^{\prime}>\lambda$ implies that $F\left(\lambda^{\prime}\right)<F(\lambda)$;

(iii) $v(P)=\operatorname{Sup}\{\lambda \mid F(\lambda)>0\}=\operatorname{Inf}\{\lambda \mid F(\lambda)<0\}$

(iv) $F(\lambda)=0$ if and only if $\lambda=v(\mathrm{P})$ and (P) has an optimal solution; then the optimal solutions $\left(x_{\lambda}, \lambda=v(\mathrm{P})\right)$ of $\left(\mathrm{P}_{\lambda}\right)$ generate the optimal solutions of $(\mathrm{P})$;

(v) $F$ is upper semicontinuous on $R$, and $\lambda=v(\mathrm{P})$ implies that $F(\lambda) \geqslant 0$; furthermore, if the set $\{x \mid C x \leqslant \gamma, x \geqslant 0\}$ is compact, then $F$ is finite and continuous on $R$.

Proof. (i) The result follows immediately from assumption $\mathrm{H}_{1}$.

(ii) Since $F(\lambda)>-\infty$ there exists an optimal solution $\left(x_{\lambda}, t_{\lambda}\right)$ of the linear program $\left(\mathrm{P}_{\lambda}\right)$. Hence

$$
\left(A x_{\lambda}+\alpha\right)-\lambda\left(B x_{\lambda}+\beta\right) \leqslant t_{\lambda} e, \quad C x_{\lambda} \leqslant \gamma, x_{\lambda} \geqslant 0 .
$$

Furthermore $t_{\lambda}=F(\lambda)$. Obviously (3.1) can be written as

$$
\left(A x_{\lambda}+\alpha\right)-\lambda^{\prime}\left(B x_{\lambda}+\beta\right)+\left(\lambda^{\prime}-\lambda\right)\left(B x_{\lambda}+\beta\right) \leqslant i_{\lambda} e .
$$

Referring to assumption $\mathrm{H}_{2},\left(B x_{\lambda}+\beta\right)>0$, and then there exists $\delta>0$ such that

$$
\left(\lambda^{\prime}-\lambda\right)\left(B x_{\lambda}+\beta\right) \geqslant \delta e .
$$

Therefore

$$
\left(A x_{\lambda}+\alpha\right)-\lambda^{\prime}\left(B x_{\lambda}+\beta\right) \leqslant\left(t_{\lambda}-\delta\right) e .
$$

Since $\left(x_{\lambda}, t_{\lambda}-\delta\right)$ is a feasible solution of $\left(\mathrm{P}_{\lambda^{\prime}}\right)$

$$
F\left(\lambda^{\prime}\right) \leqslant t_{\lambda}-\delta=F(\lambda)-\delta<F(\lambda) \text {. }
$$

(iii) First let us show that

$$
F(\lambda) \leqslant 0 \text { implies } \lambda \geqslant v(\mathrm{P}) \text {. }
$$

If $F(\lambda) \leqslant 0$, then there exists a pair $\left(x_{\lambda}, t_{\lambda}\right)$ such that

$$
\left(A x_{\lambda}+\alpha\right)-\lambda\left(B x_{\lambda}+\beta\right) \leqslant t_{\lambda} e, \quad C x_{\lambda} \leqslant \gamma, x_{\lambda} \geqslant 0, t_{\lambda} \leqslant 0 .
$$


Therefore $\left(A x_{\lambda}+\alpha\right) \leqslant \lambda\left(B x_{\lambda}+\beta\right)$, and thus $\left(x_{\lambda}, \lambda\right)$ is a feasible solution of $(\mathrm{P})$. Hence $v(\mathrm{P}) \leqslant \lambda$.

Now let us show that

$$
\lambda>v(\mathbf{P}) \text { implies } F(\lambda)<0 .
$$

If $\lambda>v(\mathrm{P})$, then there exists a pair $\left(x_{\lambda^{\prime}}, \lambda^{\prime}\right), \lambda>\lambda^{\prime}>v(\mathrm{P})$, such that

$$
\left(A x_{\lambda^{\prime}}+\alpha\right) \leqslant \lambda^{\prime}\left(B x_{\lambda^{\prime}}+\beta\right), \quad C x_{\lambda} \leqslant \gamma, x_{\lambda} \geqslant 0 .
$$

Since $\left(B x_{\lambda^{\prime}}+\beta\right)>0$ by $\mathrm{H}_{2}$,

$$
\left(A x_{\lambda^{\prime}}+\alpha\right) \leqslant \lambda^{\prime}\left(B x_{\lambda^{\prime}}+\beta\right) \leqslant \lambda\left(B x_{\lambda^{\prime}}+\beta\right) .
$$

Then the pair $x_{\lambda^{\prime}}$ and $t=0$ is a feasible solution of $\left(\mathrm{P}_{\lambda}\right)$, and hence $F(\lambda) \leqslant 0$. Notice that the same argument can be used to show that $F\left(\lambda^{\prime}\right) \leqslant 0$. Now assume $F(\lambda)=0$. The monotonicity of $F$ implies that $F\left(\lambda^{\prime}\right) \geqslant 0$, and thus $F\left(\lambda^{\prime}\right)=0$. However $F(\lambda)=0$ together with $F\left(\lambda^{\prime}\right)=0$ contradict (ii). Thus $F(\lambda)<0$.

Finally, (3.3) and the converse of (3.2) imply that

$$
v(\mathbf{P})=\operatorname{Sup}\{\lambda \mid F(\lambda)>0\}=\operatorname{Inf}\{\lambda \mid F(\lambda)<0\} .
$$

(iv) Assume that $F(\lambda)=0$. Then from (3.2) and (3.3) it follows that $\lambda=v(\mathrm{P})$. Also, the linear program $\left(\mathrm{P}_{\lambda}\right)$ has an optimal solution $\left(x_{\lambda}, t_{\lambda}\right)$ where $t_{\lambda}=0$. Thus $x_{\lambda}$ is a feasible solution of $(\mathrm{P})$ which is optimal since $\lambda=v(\mathrm{P})$.

Conversely, assume that $\lambda=v(\mathrm{P})$ and that (P) has an optimal solution $\bar{x}$. Then the pair $\left(\bar{x}, t_{\lambda}\right)$ where $t_{\lambda}=0$ is a feasible solution of $\left(\mathrm{P}_{\lambda}\right)$. Hence $F(\lambda) \leqslant 0$.

If $F(\lambda)<0$, then there exists a pair $\left(x_{\lambda}, t_{\lambda}\right)$ where $t_{\lambda}<0$ such that

$$
\left(A x_{\lambda}+\alpha\right)-\lambda\left(B x_{\lambda}+\beta\right) \leqslant t_{\lambda} e, \quad C x_{\lambda} \leqslant \gamma, x_{\lambda} \geqslant 0 .
$$

Since $\left(B x_{\lambda}+\beta\right)>0$ and $t_{\lambda}<0$ there exists a scalar $\delta<0$ such that $\delta\left(B x_{\lambda}+\beta\right)>t_{\lambda} e$. Hence

$$
\left(A x_{\lambda}+\alpha\right) \leqslant(\lambda+\delta)\left(B x_{\lambda}+\beta\right)
$$

and the pair $\left(x_{\lambda}, \lambda+\delta\right)$ is a feasible solution of $(\mathrm{P})$. Thus $v(\mathrm{P}) \leqslant \lambda+\delta<\lambda$, a contradiction. Therefore $F(\lambda)=0$.

(v) Obviously an equivalent formulation for $\left(\mathrm{P}_{\lambda}\right)$ is

$$
F(\lambda)=\operatorname{Inf}_{x}\left\{\operatorname{Max}_{1 \leqslant i \leqslant p}\left\{(A x+\alpha)_{i}-\lambda(B x+\beta)_{i}\right\} \mid C x \leqslant \gamma, x \geqslant 0\right\} .
$$

Clearly the function $\operatorname{Max}_{1 \leqslant i \leqslant p}\left\{(A x+\alpha)_{i}-\lambda(B x+\beta)_{i}\right\}$ is continuous in $(x, \lambda)$. Therefore $F$ is upper semicontinuous in $\lambda$ and continuous if $\{x=C x \leqslant \gamma, x \geqslant 0\}$ is compact. Furthermore, since $F$ is upper semicontinuous, (iii) implies that $F(\lambda) \geqslant 0$ for $\lambda \mid v(\mathrm{P})$. 
From the duality theory of linear programming, it follows that the dual of $\left(\mathrm{P}_{\lambda}\right)$ takes the form

$\left(D_{\lambda}\right)$

$$
\begin{aligned}
F(\lambda)=\operatorname{Sup}_{u^{*}, w^{*}}\left\{-\lambda\left\langle u^{*}, \beta\right\rangle+\left\langle u^{*}, \alpha\right\rangle-\left\langle w^{*}, \gamma\right\rangle \mid\right. \\
\left.\quad \lambda B^{\mathrm{T}} u^{*} \leqslant A^{\mathrm{T}} u^{*}+C^{\mathrm{T}} w^{*},\left\langle e, u^{*}\right\rangle=1, w^{*} \geqslant 0, u^{*} \geqslant 0\right\} .
\end{aligned}
$$

Furthermore if $F(\lambda)>-\infty$ then $\left(D_{\lambda}\right)$ has at least one optimal solution.

\section{4. $v(P)$ finite}

Let us now study the relationship between $(\mathbf{P})$ and $(\overline{\mathbf{D}})$ when $v(\mathbf{P})$ is finite.

\subsection{Existence of optimal solutions}

Theorem 4.1. If assumptions $\mathrm{H}_{1}$ and $\mathrm{H}_{2}$ hold, then $v(\mathrm{P})>-\infty$ implies that $(\overline{\mathrm{D}})$ has an optimal solution.

Proof. According to part (v) in Theorem 3.1, $F(\lambda) \geqslant 0$ for $\lambda=v(\mathrm{P})$. Since $F(\lambda)$ is finite the linear program $\left(D_{\lambda}\right)$ has an optimal solution. This is also an optimal solution for $(\bar{D})$ since $F(\lambda) \geqslant 0$, and $\lambda=v(\mathbf{P})$ is the optimal value of $(\overline{\mathbf{D}})$.

Theorem 4.2. If assumptions $\mathrm{H}_{1}$ and $\mathrm{H}_{2}$ hold, then the primal problem (P) has an optimal solution if and only if for all optimal solutions $\left(v(\mathrm{P}), u^{*}, w^{*}\right)$ of $(\overline{\mathrm{D}})$ one has

$$
v(\mathrm{P})\left\langle u^{*}, \beta\right\rangle=\left\langle u^{*}, \alpha\right\rangle-\left\langle w^{*}, \gamma\right\rangle .
$$

Proof. Assume that $(\mathrm{P})$ has an optimal solution. Then in view of part (iv) of Theorem 3.1, $F(\lambda)=0$ if $\lambda=v(\mathrm{P})$. Hence, for all optimal solutions $\left(u^{*}, w^{*}\right)$ of $\left(\mathrm{D}_{\lambda}\right)$,

$$
-v(\mathrm{P})\left\langle u^{*}, \beta\right\rangle+\left\langle u^{*}, \alpha\right\rangle-\left\langle w^{*}, \gamma\right\rangle=0 .
$$

But an optimal solution of $\left(D_{\lambda}\right)$ corresponds to an optimal solution of $(\overline{\mathrm{D}})$, and vice versa.

Conversely, if the property holds for all optimal solutions of $(\overline{\mathrm{D}})$, then $F(\lambda)=0$. Hence, referring to part (iv) of Theorem 3.1, (P) has an optimal solution.

This theorem determines a necessary and sufficient condition for the existence of a solution for primal $(\mathrm{P})$. Note that the condition involves all the solutions for the dual $(\overline{\mathrm{D}})$.

\subsection{Complementary slackness}

In this section complementarity between variables in one problem (Primal or Dual) and associated constraints in the other (Dual or Primal) is derived. This information might be useful to determine a solution of the Primal (Dual) whenever a solution for the Dual (Primal) is known. 
Assume that $v(\mathrm{P})>-\infty, \bar{x}$ is a solution for the Primal $(\mathrm{P})$, and $\left(\bar{u}^{*}, \bar{w}^{*}\right)$ is a solution for the Dual ( $\overline{\mathrm{D}})$. Then the following relations are verified

$$
\begin{aligned}
& C \bar{x}-\gamma \leqslant 0, \\
& A \bar{x}+\alpha-v(\mathrm{P}) B \bar{x}-v(\mathrm{P}) \beta \leqslant 0, \\
& v(\mathrm{P})\left\langle\bar{u}^{*}, \beta\right\rangle-\left\langle\bar{u}^{*}, \alpha\right\rangle+\left\langle\bar{w}^{*}, \gamma\right\rangle \leqslant 0, \\
& v(P) B^{\mathrm{T}} \ddot{u}^{*}-A^{\mathrm{T}} \bar{u}^{*}-C^{\mathrm{T}} \bar{w}^{*} \leqslant 0, \\
& \quad \bar{x} \geqslant 0, \bar{u}^{*} \geqslant 0, \bar{u}^{*} \neq 0, \bar{w}^{*} \geqslant 0 .
\end{aligned}
$$

After multiplying (4.1) by $\bar{w}^{*},(4.2)$ by $\bar{u}^{*}$, and (4.4) by $\bar{x}$, one obtains

$$
\begin{aligned}
& \left\langle C^{\mathrm{T}} \bar{w}^{*}, \bar{x}\right\rangle-\left\langle\bar{w}^{*}, \gamma\right\rangle \leqslant 0, \\
& \left\langle A^{\mathrm{T}} \bar{u}^{*}, \bar{x}\right\rangle+\left\langle\bar{u}^{*}, \alpha\right\rangle-v(\mathrm{P})\left\langle B^{\mathrm{T}} \bar{u}^{*}, \bar{x}\right\rangle-v(\mathrm{P})\left\langle\bar{u}^{*}, \beta\right\rangle \leqslant 0, \\
& v(\mathrm{P})\left\langle B^{\mathrm{T}} \bar{u}^{*}, \bar{x}\right\rangle-\left\langle A^{\mathrm{T}} \bar{u}^{*}, \bar{x}\right\rangle-\left\langle C^{\mathrm{T}} \bar{w}^{*}, \bar{x}\right\rangle \leqslant 0 .
\end{aligned}
$$

Consider the sum of (4.3), (4.6) and (4.7):

$$
-\left\langle C^{\mathrm{T}} \bar{w}^{*}, \bar{x}\right\rangle+\left\langle\bar{w}^{*}, \gamma\right\rangle \leqslant 0 .
$$

Thus (4.8) and (4.5) imply that equality holds, i.e.

$$
-\left\langle C^{\mathrm{T}} \bar{w}^{*}, \bar{x}\right\rangle+\left\langle\bar{w}^{*}, \gamma\right\rangle=0 .
$$

From this we see that

$$
\text { if } \bar{w}_{i}^{*}>0 \text { then }(C \bar{x}-\gamma)_{i}=0 .
$$

Furthermore the construction of $(4.8)$ shows that in view of $(4.9)$ equality holds in (4.3), (4.6) and (4.7). In particular we have

$$
\left\langle A^{\mathrm{T}} \bar{u}^{*}, \bar{x}\right\rangle+\left\langle\bar{u}^{*}, \alpha\right\rangle-v(P)\left\langle B^{\mathrm{T}} \bar{u}^{*}, \bar{x}\right\rangle-v(\mathrm{P})\left\langle\bar{u}^{*}, \beta\right\rangle=0
$$

and

$$
v(\mathbf{P})\left\langle B^{\mathrm{T}} \bar{u}^{*}, \bar{x}\right\rangle-\left\langle A^{\mathrm{T}} \bar{u}^{*}, \bar{x}\right\rangle-\left\langle C^{\mathrm{T}} \bar{w}^{*}, \bar{x}\right\rangle=0 .
$$

\section{5. $v(P)$ infinite}

Now conditions in terms of the matrices $A, B, C$ are derived for $v(\mathrm{P})$ to be infinite.

Lemma 5.1. Assume that assumptions $\mathrm{H}_{1}$ and $\mathrm{H}_{2}$ hold. Then $v(\mathrm{P})=-\infty$ if and only if for all scalar $\tau>0$ there exists a vector $y \in \mathbb{R}^{n}, y \geqslant 0$, such that $C y \leqslant 0$, $A y+\tau B y<0$ and $B y \geqslant 0$.

Proof. Let us first derive the result when $\mathrm{H}_{1}$ and $\mathrm{H}_{3}$ hold. Referring to the definition of $(\overline{\mathrm{D}})$, it follows that $v(\mathrm{P})=-\infty$ if and only if for all $u^{*} \geqslant 0, u^{*} \neq 0, w^{*} \geqslant 0$ there 
exists an index $1 \leqslant j \leqslant n$ such that $\left\langle u^{*}, b_{\cdot j}\right\rangle=0$ and $\left\langle u^{*}, a_{\cdot j}\right\rangle+\left\langle w^{*}, c_{\cdot j}\right\rangle<0$. This is equivalent to requiring that, for all $u^{*} \geqslant 0, u^{*} \neq 0, w^{*} \geqslant 0$ and for all scalars $\tau>0$, the vector

$$
A^{\mathrm{T}} u^{*}+\tau B^{\mathrm{T}} u^{*}+C^{\mathrm{T}} w^{*}
$$

is not nonnegative. Hence $v(\mathrm{P})=-\infty$ if and only if for all $u^{*} \geqslant 0, w^{*} \geqslant 0$ and for all $\tau>0$

$$
u^{*} \neq 0 \text { implies } A^{\mathrm{T}} u^{*}+\tau B^{\mathrm{T}} u^{*}+C^{\mathrm{T}} w^{*} \text { is not nonnegative, }
$$

or, equivalently,

Let $e=[1,1, \ldots, 1]^{\mathrm{T}} \in \mathbb{R}^{\prime \prime}$. Now (5.2) is true if and only if 0 is the optimal value for the problem

$$
\begin{array}{ll}
\operatorname{Max} & \left\langle e, u^{*}\right\rangle \\
\text { s.t. } & -A^{\mathrm{\top}} u^{*}-\tau B^{\top} u^{*}-C^{\mathrm{T}} w^{*} \leq 0, \\
& u^{*} \geqslant 0, w^{*} \geqslant 0 .
\end{array}
$$

Then, from a duality result in linear programming, it follows that $v(\mathbf{P})=-\infty$ if and only if the dual of (5.3) has an optimal solution with value 0 , i.e. if and only if for all $\tau>0$ there exists a vector $y \in \mathbb{R}^{\prime \prime}, y \geqslant 0$, such that

$$
C y \leqslant 0, \quad A y+\tau B y \leqslant-e<0 .
$$

Also, $B y \geqslant 0$ is a consequence of $\mathrm{H}_{3}$.

Now let us extend this result to cases where assumption $\mathrm{H}_{2}$ replaces $\mathrm{H}_{3}$. If $\mathrm{H}_{2}$ holds, then there exists a scalar $k>0$ such that

$$
B x+\beta \geqslant k e \text { for all } x \geqslant 0 \text { and } C x \leqslant \gamma .
$$

Let us introduce the new variable

$$
r=B x+\beta-k e .
$$

Then $(\mathrm{P})$ can be written as

$$
\operatorname{Inf}_{\lambda, x, r}\{\lambda \mid A x+\alpha \leqslant \lambda(r+k e), C x \leqslant \gamma, B x-r=k e-\beta, r \geqslant 0, x \geqslant 0\} .
$$

Now define the matrices

$$
\begin{aligned}
& \hat{A}=[A, 0], \quad \text { a } p \times(n+p) \text { matrix, } \\
& \hat{B}=[0, I], \quad \text { a } p \times(n+p) \text { matrix, } \\
& \hat{C}=\left[\begin{array}{rr}
C, & 0 \\
B, & -I \\
-B, & I
\end{array}\right], \quad \text { an }(m+2 p) \times(n+p) \text { matrix, }
\end{aligned}
$$


and the vectors $\hat{\xi}^{\mathrm{T}}=\left[x^{\mathrm{T}}, r^{\mathrm{T}}\right], \hat{\alpha}=\alpha, \hat{\beta}=k e$ and

$$
\hat{\gamma}=\left[\begin{array}{c}
\gamma \\
k e-\beta \\
-k e+\beta
\end{array}\right] \in \mathbb{R}^{m+2 p}
$$

Then $(\mathrm{P})$ can be formulated as

$$
\operatorname{Inf}_{\lambda, x, r}\{\lambda \mid \hat{A} \xi+\alpha \leqslant \lambda(\hat{B} \xi+\hat{\beta}), \hat{C} \xi \leqslant \hat{\gamma}, \xi \geqslant 0\} .
$$

Now $\hat{B}$ and $\hat{\beta}$ satisfy assumption $\mathrm{H}_{3}$. Hence using the first part of the proof, it follows that $v(\mathrm{P})=-\infty$ if and only if for all scalar $\tau>0$ there exists a vector $\Pi \in \mathbb{R}^{n+n}$, $\Pi \geqslant 0$, such that $\hat{C} \Pi \leqslant 0$ and $\hat{A} \Pi+\tau \hat{B} \Pi<0$. Now, let $\Pi^{\mathrm{T}}=\left[y^{\mathrm{T}}, s^{\mathrm{T}}\right]$ where $y \in \mathbb{R}^{n}$ and $s \in \mathbb{R}^{p}$. Then the condition reduces to the following one: for all scalar $\tau>0$ there exists vectors $y \in \mathbb{R}^{n}, s \in \mathbb{R}^{p}, y \geqslant 0, s \geqslant 0$ such that

$$
C y \leqslant 0, \quad B y=s, \quad A y+\tau s<0 .
$$

Hence the condition reduces to $C y \leqslant 0, A y+\tau B y<0$ and $B y \geqslant 0$.

Now, this result is used to determine necessary or sufficient conditions to have $v(\mathbf{P})=-\infty$.

Theorem 5.2. Assume that assumptions $\mathrm{H}_{1}$ and $\mathrm{H}_{2}$ hold.

(i) If $v(\mathrm{P})=-\infty$, then there exists a vector $y \in \mathbb{R}^{n}, y \geqslant 0, y \neq 0$, such that $C y \leqslant 0$, $B y=0$, and $A y \leqslant 0$.

(ii) If there exists a vector $y \in \mathbb{R}^{n}, y \geqslant 0, y \neq 0$ such that $C y \leqslant 0, B y=0$ and $A y<0$, then $v(\mathrm{P})=-\infty$.

Proof. (i) Assume that $v(\mathrm{P})=-\infty$. From Lemma 5.1 it follows that for all $\tau>0$ there exists a vector $y \in \mathbb{R}^{\prime \prime}$, depending on $\tau$, such that $y \geqslant 0, y \neq 0, C y \leqslant 0$ and $A y+\tau B y<0$. Since these systems are homogeneous, $y$ can be assumed to belong to the set

$$
\Gamma=\left\{z \in E^{n} \mid z \geqslant 0 \text { and } \sum_{i=1}^{n} z_{i}=1\right\} .
$$

Now, let us consider a sequence $\left\{\tau_{k}\right\}$ converging to $+\infty$, and denote by $\left\{y^{k}\right\}$ the corresponding sequence in $\Gamma$ such that $C y^{k} \leqslant 0, A y^{k}+\tau_{k} B y^{k}<0$ and $B y^{k} \geqslant 0$. Since $\Gamma$ is compact, there exists a vector $\bar{y} \in \Gamma$ and a subsequence $\left\{\tau_{k_{i}}\right\}$ converging to $+\infty$ such that $\left\{y^{k_{i}}\right\}$ converges to $\bar{y}$. Clearly $\left\{C y^{k_{i}}\right\}$ converges to $C \bar{y},\left\{A y^{k_{i}}\right\}$ to $A \bar{y},\left\{B y^{k_{i}}\right\}$ to $B \bar{y}$, and $B \bar{y} \geqslant 0$. It follows that $C \bar{y} \leqslant 0, A \bar{y} \leqslant 0$ and $B \bar{y}=0$.

(ii) Trivially, if such a vector exists, then it satisfies the condition in Lemma S.1.

The conditions in part (i) of Theorem 5.2 are necessary but not sufficient to have $v(\mathrm{P})=-\infty$. Indeed, consider the following example where $m=0, n=p=1, \alpha_{1}=0$, 
$\beta_{1}=1, A$ and $B$ are a $1 \times 1$ nul matrix. Then $v(\mathrm{P})=0$ whereas the conditions are satisfied.

Also, the conditions in part (ii) of Theorem 5.2 are sufficient but not necessary to have $v(\mathrm{P})=-\infty$. Indeed, consider the following example where $m=0, n=3$, $p=2$,

$$
A=\left[\begin{array}{rrr}
0 & -1 & 0 \\
-1 & 0 & 0
\end{array}\right] \text { and } B=\left[\begin{array}{lll}
0 & 0 & 1 \\
0 & 1 & 0
\end{array}\right]
$$

Clearly the conditions in part (ii) of Theorem 5.2 are not satisfied. But on the other hand

$$
A y+\tau B y=\left[\begin{array}{l}
-y_{2}+\tau y_{3} \\
-y_{1}+\tau y_{2}
\end{array}\right]
$$

and taking $y_{1}=1, y_{3}=0$, and $y_{2}=1 / 2 \tau$ for $\tau>0$, the conditions of Lemma 5.1 are satisfied, and so $v(\mathrm{P})=-\infty$.

It is worth noticing the similarity of this result to the alternative theorems analyzing the existence of solutions to systems of linear inequalities (see Mangasarian [14]).

For the single ratio problem $(p=1)$ the duality relations in section 4 and 5 were derived by Schaible in $[17,18,20]$.

\section{A special case}

Now, assume that $H_{1}$ holds and that $\mathrm{H}_{2}$ is replaced by $\mathrm{H}_{3}$. Obviously, $\mathrm{H}_{3}$ implies $\mathrm{H}_{2}$.

Then an equivalent formulation for problem $(\overline{\mathbf{D}})$ is as follows:

$$
\sup _{\substack{u^{*} \geqslant 0, w^{*} \geq 0 \\ u^{*} \neq 0}}\left\{\operatorname{Min}\left\{\frac{\left\langle u^{*}, \alpha\right\rangle-\left\langle w^{*}, \gamma\right\rangle}{\left\langle u^{*}, \beta\right\rangle}, \operatorname{Min}_{1 \leqslant j \leqslant n}\left\{\frac{\left\langle u^{*}, a_{, j}\right\rangle+\left\langle w^{*}, c_{. j}\right\rangle}{\left\langle u^{*}, b_{j}\right\rangle}\right\}\right\}\right\}
$$

with the convention that

$$
\frac{\rho}{0}= \begin{cases}+\infty & \text { if } \rho \geqslant 0 \\ -\infty & \text { if } \rho<0\end{cases}
$$

Hence under condition $\mathrm{H}_{3}$ the dual of $(\mathrm{P})$ is again a generalized linear fractional programming problem. This version of the dual in case $p=1$ was introduced by Tammer in [23] (see also [20]).

In Section 4.2 complementary slackness results were derived. Under the assumption $\mathrm{H}_{3}$ eqs. (4.10) and (4.11) then imply: if $\bar{u}_{k}^{*}>0$ then

$$
v(\mathbf{P})=\frac{\left\langle a_{k \cdot}^{\mathrm{T}}, \bar{x}\right\rangle+\alpha_{k}}{\left\langle b_{k}^{\mathrm{T}}, \bar{x}\right\rangle+\beta_{k}}=\operatorname{Max}_{i \leqslant i \leqslant p}\left\{\frac{\left\langle a_{i}^{\mathrm{T}}, \bar{x}\right\rangle+\alpha_{i}}{\left\langle b_{i}^{\mathrm{T}}, \bar{x}\right\rangle+\beta_{i}}\right\}
$$


and if $\tilde{x}_{l}>0$, then

\section{References}

$$
v(\mathrm{P})=\frac{\left\langle\bar{u}^{*}, a_{\cdot l}\right\rangle+\left\langle\bar{w}^{*}, c_{. l}\right\rangle}{\left\langle\bar{u}^{*}, b_{. l}\right\rangle}=\operatorname{Min}_{1 \leq i \leq n}\left\{\frac{\left\langle\bar{u}^{*}, a_{\cdot j}\right\rangle+\left\langle\bar{w}^{*}, c_{\cdot j}\right\rangle}{\left\langle\bar{u}^{*}, b_{\cdot j}\right\rangle}\right\} .
$$

[1] D.J. Ashton and D.R. Atkins, "Multicriteria programming for financial planning", Journal of the Operational Research Society 30 (1979) 259-270.

[2] I. Barrodale, "Best rational approximation and strict quasi-convexity", SIAM Journal of Numerical Analysis 10 (1973) 8-12.

[3] A. Charnes and W.W. Cooper, "Programming with linear fractional functionals", Naval Research Logistics Quarterly 9 (1962) 181-186.

[4] A. Charnes and W.W. Cooper, "Goal programming and multi-objective optimization (Part I)", European Journal of Operational Research 1 (1977) 39-54.

[5] A. Charnes, L. Cox and M. Lane, "A Note on the redesigning of a rate structure for allocation of state funds to educational institutions", Working Paper 70-49 of Project GUM, University of Texas at Austin (Austin, Texas, 1970).

[6] J.P. Crouzeix, "Contributions à l'étude des fonctions quasiconvexes", Doctoral Thesis, Université de Clermont (Clermont, France, 1977).

[7] J.P. Crouzeix, "A duality framework in quasiconvex programming", in: S. Schaible and W.T. Ziemba, eds., Generalized Concavity in Optimization and Economics (Academic Press, New York, 1981) pp. 207-225.

[8] W. Dinkelbach, "On nonlinear fractional programming", Management Science 13 (1967) 492 -498.

[9] J. Flachs, "Global saddle-point duality for quasi-concave programs, II", Mathematical Programming 24 (1982) 326-345.

[10] E.G. Golstein, Theory of convex programming, Translations of mathematical monographs 36 (American Mathematical Society, Providence, Rhode Island, 1972).

[11] R. Jagannathan, "On some properties of programming problems in parametric form pertaining to fractional programming", Management Science 12 (1966) 609-615.

[12] R. Jagannathan and S. Schaible. "Duality in generalized fractional programming via Farkas Lemma", Journal of Optimization Theory and Applications, to appear.

[13] J.S.H. Kornbluth, "A survey of goal programming", OMEGA 1 (1973) 193-205.

[14] O.L. Mangasarian, Nonlinear programming (McGraw-Hill, New York, 1969).

[15] U. Passy and A. Keslassy, "Pseudo duality and duality for explicitly quasiconvex functions". Mimeograph Series No. 249. Faculty of Industrial Engineering and Management, Technion (Haifa, Israel, 1979).

[16] G.S. Rubinshtein, "Duality in mathematical programming and some problems of convex analysis", (English translation) Russian mathematical surveys 25 (1970) 171-200.

[17] S. Schaible, "Fractional programming: transformations, duality and algorithmic aspects", Technical Report 73-9, Department of Operations Research, Stanford University (Stanford, CA, 1973).

[18] S. Schaible, "Fractional programming I, Duality", Management Science 22 (1976) 858-867.

[19] S. Schaible, "Duality in fractional programming: a unified approach", Operations Research 24 (1976) 452-461.

[20] S. Schaible, Analyse und Anwendungen von Quotientenprogrammen (Hain-Verlag, Meisenheim, 1978).

[21] S. Schaible, "A survey of fractional programming", in: S. Schaible and W.T. Ziemba, eds., Generalized concavity in optimization and economics (Academic Press. New York, 1981) pp. 417-440.

[22] S. Schaible, "Bibliography in fractional programming", Zeitschrift für Operations Research 26 (7) (1982).

[23] E.C. Tammer, "Dualitätstheorie für hyperbolische und stückweise-lineare konvexe Optimierungsprobleme", Mathematische Operationenforschung und Statistik 5 (1974) 93-108.

[24] R. Vogt, "A corporate strategy for realizing equal employment opportunity", Behavioral and Social Accounting, to appear.

[25] J. von Neumann, "A model of general economic equilibrium", Review of Economic Studies 13 (1945) $1-9$. 